\title{
Indolent lymphoma with composite histology and simultaneous transformation at initial diagnosis exhibit clinical features similar to de novo diffuse large B-cell Iymphoma
}

\author{
Hanno Witte ${ }^{1, *}$, Harald Biersack ${ }^{1, *}$, Svenja Kopelke $^{1}$, Dirk Rades ${ }^{2}$, Hartmut Merz ${ }^{3}$, \\ Veronica Bernard ${ }^{3}$, Hendrik Lehnert ${ }^{1}$ and Niklas Gebauer ${ }^{1}$ \\ ${ }^{1}$ Department of Hematology and Oncology, University Hospital of Schleswig-Holstein, Campus Luebeck, Luebeck, Germany \\ ${ }^{2}$ Department of Radiation Oncology, University Hospital of Schleswig-Holstein, Campus Luebeck, Luebeck, Germany \\ ${ }^{3}$ Hämatopathologie Lübeck, Reference Center for Lymph Node Pathology and Hematopathology, Lübeck, Germany \\ *These authors have contributed equally to this work \\ Correspondence to: Niklas Gebaver, email: Niklas.Gebaver@uksh.de \\ Keywords: transformed indolent lymphoma; composite histology; de novo diffuse large B-cell Lymphoma; cell-of-origin; prognosis \\ Received: December 07, $2017 \quad$ Accepted: March 02, $2018 \quad$ Published: April 13, 2018 \\ Copyright: Witte et al. This is an open-access article distributed under the terms of the Creative Commons Attribution License 3.0 \\ (CC BY 3.0), which permits unrestricted use, distribution, and reproduction in any medium, provided the original author and source \\ are credited.
}

\section{ABSTRACT}

While various studies characterized clinical and prognostic properties of de novo diffuse large $B$-cell lymphoma (DLBCL) and transformed indolent lymphomas, the clinicopathological features of indolent lymphoma and simultaneous secondary transformation upon initial diagnosis (ssDLBCL) are insufficiently established.

Between 2010 and 2017, 247 consecutive patients admitted to our institution and treated for DLBCL were investigated for composite histology of sSDLBCL-type. Upon systematical histopathological evaluation composite histology was identified in $22 / 247$ cases $(8.9 \%)$.

The predominant histology of the underlying indolent lymphoma was follicular lymphoma of variable grading (I-IIIA; $81.8 \%$ ) whereas marginal zone lymphoma represented a minor sub group (18.2\%). Clinicopathological investigation revealed a high degree of concordance between SSDLBCL and de novo DLBCL upon initial diagnosis and clinical courses were shown to be strikingly similar. The predominant fraction of SSDLBCL were germinal center derived lymphomas (GCB-type) with a trend towards a superior outcome compared with non-GCB-type ssDLBCL. Additionally, we demonstrate a significant adverse prognostic impact of an underlying indolent lymphoma component other than follicular-type lymphoma (e.g. marginal zone lymphoma). Moreover, the frequency of double-hit (DHL) or double-expressor lymphomas (DEL) appears to be low.

Our findings provide substantial insight into the behavior of SSDLBCL, highlight the ramifications of the concurrent high-grade fraction within indolent lymphomas and underline therapeutic efficacy of R-CHOP type immunochemotherapy in the majority of ssDLBCL patients.

\section{INTRODUCTION}

The development of aggressive non-Hodgkin's lymphoma (typically DLBCL) in patients with underlying indolent lymphoma is commonly referred to as transformation $[1,2]$. Diffuse large B-cell lymphoma (DLBCL) is the most common type of non-Hodgkin's lymphoma (NHL), and its incidence is closely correlated with increasing age [3]. In most studies this entity is subdivided into the categories of de novo DLBCL and 
transformed high-grade lymphomas with a preceding history of indolent (most commonly follicular and marginal zone derived) lymphoma. Studies predating the introduction of immunochemotherapy found follicular lymphoma that transformed to DLBCL (tFL) as well as other secondary high-grade B-cell lymphomas to portend a dismal prognosis with a median overall survival (OS) of approximately two years [4, 5]. Common indicators of transformation in primarily indolent NHL are raised LDH level, alternative biochemical surrogate markers for elevated cellular turnover (e.g. hypercalcemia, hyperuricaemia), rapid nodal/extranodal growth, swiftly diminishing performance status or increasing B symptoms as well as newly emerging sites of extranodal manifestation [6-9]. Clinical and prognostic data on indolent lymphoma with composite histology and simultaneous secondary transformation at initial diagnosis (ssDLBCL) or "transformed lymphoma at diagnosis" in the era of immunochemotherapy remain sparse as most prior studies on both indolent lymphoma as well as DLBCL neglected these patients. Despite the deficiency of prospective data in this group of patients, immunochemotherapy first-line treatment of aggressive lymphoma-type (e.g. Rituximab, Cyclofosfamide, Doxorubicine, Vincristin \& Prednisolone (R-CHOP)) occasionally followed by high-dose chemotherapy and autologous stem cell transplantation (ASCT) has emerged as the therapeutic strategy of choice [10-12]. Only very recently a large-scale case study by Magnano et al. was published concluding that the outcome of ssDLBCL patients may not be worse than that of de novo DLBCL questioning the need for widely applied intensification with ASCT following induction by immunochemotherapy [13]. The central aim of the current retrospective singlecenter study was a clinicopathological characterization of an extended cohort of ssDLBCL patients, incorporating concurrent underlying indolent lymphoma histologies of all types (e.g. including marginal zone derived lymphomas).

\section{RESULTS}

\section{Histopathology}

The histological distribution of the concurrent indolent component was: 5 FL grade $1 / 2(22.7 \%), 13$ FL grade $3 \mathrm{~A}(59.1 \%)$ and 4 marginal zone lymphoma (18.2\%). Immunohistochemical data on BCL2, BCL6 and MYC expression as well as Cell of origin (COO) as determined immunohistochemically via the longestablished algorithm by Hans et al. showing a germinal center type expression pattern (GCB-type) in 17/22 patients are briefly summarized in Table 1. Data on COO partitioned by underlying indolent lymphoma subtype are summarized in Supplementary Table 2. FISH studies were available in only 4/22 ssDLBCL patients and are summarized in Supplementary Table 3. Moreover the extent of the DLBCL component within the diagnostic sample (ranging from $30-90 \%$ ) alongside the type of biopsy specimen as well as other complementary data derived from histopathological evaluation are summarized in Supplementary Tables 4 and 5. Cytology of malignant bone marrow infiltrates in cases of ssDLBCL upon initial diagnosis (4/22) was composed of small cells in $3 / 3$ ssDLBCL with FL-type component whereas the case of MZL-type ssDLBCL with bone marrow involvement (1/4) exhibited large cell infiltrates.

\section{Clinical characteristics, treatment response and outcome}

The mean age at diagnosis of the 22 evaluable patients was 61.3 (range $34-79$ years). Thirteen patients (59.1\%) were male. We found no significant disparities within the group of ssDLBCL based on type of concurrent indolent lymphoma. The vast majority of patients received R-CHOP induction therapy upon diagnosis $(19 / 22)$ while only one of the ssDLBCL patients refused any cytoreductive treatment. In addition to six cycles of R-CHOP 6/19 patients received two additional cycles of rituximab monotherapy and another four patients received rituximab maintenance therapy over the course of two years as described [14]. Median follow-up was 43.5 months for ssDLBCL patients and 41 months in the de novo control group respectively. None of the assessed clinical parameters (Age, R-IPI, ECOG PS, LDH, extranodal sites, Ann Arbor stage) differed significantly between ssDLBCL and de novo DLBCL. Prognostic survey revealed further similarities as the 2-year OS observed in the current study was $84.2 \%$ and $78.0 \%$ respectively ( $p=0,3293$; Hazard Ratio (HR) 0,6257; 95\% confidence interval (CI) 0,244 to 1,61). Moreover, the 2 -year PFS was $81.1 \%$ and $71.9 \%$ respectively $(p=0,272$; HR: 0,652; CI: 0,304 to 1,398). Clinical characteristics and therapeutic frontline approaches of all patients are summarized in Table 2 whereas composition of both study group and controls as well as survival data are depicted in Figures 1 and 2. Upon relapse a confirmatory biopsy was obtained in all cases and all but one of the ssDLBCL (4/5, $80 \%$ ) exhibited high-grade histology without signs of a concurrent indolent fraction. Within the group of ssDLBCL relapse was however associated with dismal prognosis and $3 / 5$ patients died with a median post-relapse survival of 7.5 months. Salvage treatment approaches are depicted in Supplementary Table 6. A preliminary univariate analysis followed by a Cox proportional hazard-type multivariate analysis revealed Stage III/IV $(\mathrm{p}=0.005)$ and R-IPI $(\mathrm{p}<0.0001)$ to be the only significant predictors of OS. While ECOG $>1(p=0.001)$ and R-IPI $(p=0.004)$ were the only predictors of PFS. Both multivariate analysis as well as a confirmatory propensity score matched analysis ruled out a significant impact of the presence of composite 
Table 1: Immunohistochemical data on ssDLBCL by type of underlying indolent lymphoma histology

\begin{tabular}{lcc}
\hline Antibody & FL-type ssDLBCL $(\mathbf{n}=\mathbf{1 8})$ & MZL-type ssDLBCL $(\mathbf{n}=\mathbf{4})$ \\
\hline CD10 & $16 / 18(88.9 \%)$ & $1 / 4(25 \%)$ \\
MUM-1 & $2 / 11(18.2 \%)$ & $4 / 4(100 \%)$ \\
BCL2 & $14 / 16(87.5 \%)$ & $3 / 4(75 \%)$ \\
BCL6 & $7 / 10(70 \%)$ & $3 / 3(100 \%)$ \\
MYC & $0 / 12(0)$ & $0 / 2(0)$ \\
GCB (Hans et al.) & $16 / 18(88.9 \%)$ & $1 / 4(25 \%)$ \\
Non-GCB (Hans et al. $)$ & $2 / 18(11.1 \%)$ & $3 / 4(75 \%)$ \\
Ki67 (median +/- SD) & $75(+/-17.2)$ & $70(+/-15)$ \\
\hline
\end{tabular}

ssDLBCL, DLBCL, diffuse large B-cell Lymphoma; ss, simultaneous secondary; FL, follicular lymphoma; MZL, marginal zone lymphoma; GCB, germinal center B-cell like; SD, standard deviation.

histology on OS ( $\mathrm{p}=0.644$ and $\mathrm{p}=0.705$ respectively) and PFS ( $\mathrm{p}=0.794$ and $\mathrm{p}=0.236$ respectively). Detailed results regarding the prognostic impact of clinical characteristics of OS and PFS are presented in Supplementary Table 7.

In addition we detected a significant impact of underlying indolent lymphoma histology on both OS and PFS favoring follicular lymphoma-type composite disease ( $\mathrm{p}=0,0299$; Hazard Ratio (HR) 0,03892; 95\% confidence interval (CI) 0,002 to 0,73 and $p=0,0416$; Hazard Ratio (HR) 0,$05305 ; 95 \%$ confidence interval (CI) 0,0031 to 0,895 respectively; Figure $3 \mathrm{~A}$ and $3 \mathrm{~B}$ ). Moreover, we observed a trend towards an inferior clinical outcome bordering on statistical significance for non-germinal center derived ssDLBCL according to the Hans algorithm for both OS ( $\mathrm{p}=0.0579$; Hazard Ratio (HR) 0,07; 95\% confidence interval (CI) 0,0045 to 1,093) and PFS $(\mathrm{p}=0.1218$; Hazard Ratio (HR) 0,139; $95 \%$ confidence interval (CI) 0,011 to 1,695; Figure 3C and 3D).

\section{DISCUSSION}

In the present manuscript we outline our experience of 22 patients with ssDLBCL. Aiming to minimize selection bias 247 consecutive patients diagnosed at a single institution following centralized pathology review with a lymphoproliferative disease harboring a significant DLBCL component were recruited and evaluated retrospectively.

DLBCL is the most common subtype of NHL and implementation of R- CHOP has rendered the disease curable in about $70 \%$ of patients [15]. Even more promising outcomes, yet no sustainable cures, have been achieved in the treatment of indolent lymphomas (e.g. FL \& MZL) employing immunochemotherapy [16]. The cumulative incidence of concurrent histological transformation in patients with indolent B-cell neoplasia have been incompletely evaluated. In this series depicting the clinical features and outcomes of ssDLBCL patients in the immunochemotherapy era the proportion of composite histology was 22/247 (8.9\%).

All potential features of transforming or transformed indolent lymphoma were distributed similarly among ssDLBCL and de novo DLBCL in our current study. In keeping with the observations by Wagner-Johnston et al. a considerably higher percentage of patients with composite histology with transformation at initial diagnosis was treated with R-CHOP compared with the common therapeutic approaches described for indolent lymphoma [17]. The overall survival of approximately $80 \%$ at five years of follow-up was well beyond previously reported findings in transformed indolent lymphoma [6, 18]. However, Al-Tourah et al. recently discovered that patients with limited extent transformation had a significantly better survival with a 5-year post-transformation survival of $66 \%$ compared to former reports in a population based analysis [18-20].

Apart from three rituximab-era studies that identified 5/63 (7.9\%) 40/878 (4.6\%) and 77/2734 $(2.8 \%)$ patients with tFL at the time of diagnosis, the clinical characteristics and outcomes associated with transformation at diagnosis are not well characterized [13, $17,21]$. The few studies on ssDLBCL almost exclusively included ssDLBCL of FL-type and revealed unanimously a prognosis inferior to grade III FL but similar to de novo DLBCL [22]. Younger mean age and sparse significant comorbidities may have contributed to the overall favorable prognosis of ssDLBCL in our current study. Another observation of note was that none of the 4/22 patients who received rituximab maintenance therapy showed signs of relapse at a median follow-up of 24.5 months. The limited size of the study group and duration of follow-up as well as the indistinct safety and therapeutic efficacy of rituximab maintenance in SsDLBCL require further prospective evaluation, especially in the light 
Table 2: Clinicopathological characteristics and therapeutic frontline approaches of the study group

\begin{tabular}{|c|c|c|c|}
\hline & $\operatorname{ssDLBCL}(n=22)$ & de novo DLBCL $(\mathrm{n}=166)$ & p-value \\
\hline Age (yrs.; mean + range) & $61.3(34-79)$ & $69.0(17-92)$ & 0.086 \\
\hline \multicolumn{4}{|l|}{ Sex } \\
\hline female & $9(40.9 \%)$ & $72(43.4 \%)$ & \\
\hline male & $13(59.1 \%)$ & $94(56.6 \%)$ & \\
\hline R-IPI & & & 0.3810 \\
\hline 0 & $4(18.2 \%)$ & $14(8.4 \%)$ & \\
\hline $1-2$ & $10(45.5 \%)$ & $78(47.0 \%)$ & \\
\hline$>2$ & $8(36.3 \%)$ & $74(44.6 \%)$ & \\
\hline Stage (Ann Arbor) & & & 0.4257 \\
\hline I & $4(18.2 \%)$ & $27(16.3 \%)$ & \\
\hline II & $7(31.9 \%)$ & $46(27.7 \%)$ & \\
\hline III & $6(27.2 \%)$ & $37(22.3 \%)$ & \\
\hline IV & $5(22.7 \%)$ & $56(33.7 \%)$ & \\
\hline Extranodal sites & & & 0.1987 \\
\hline 0 & $12(54.6 \%)$ & $70(42.2 \%)$ & \\
\hline $1-2$ & $9(40.9 \%)$ & $70(42.2 \%)$ & \\
\hline$>2$ & $1(4.5 \%)$ & $26(15.5 \%)$ & \\
\hline ECOG PS & & & 0.4426 \\
\hline 0 & $4(18.2 \%)$ & $34(20.5 \%)$ & \\
\hline $1-2$ & $13(59.1 \%)$ & $81(48.8 \%)$ & \\
\hline$>2$ & $5(22.7 \%)$ & $51(30.7 \%)$ & \\
\hline LDH & & & 0.4701 \\
\hline Normal & $5(22.7 \%)$ & $59(35.5 \%)$ & \\
\hline Elevated & $17(77.3 \%)$ & $107(64.5 \%)$ & \\
\hline CNS involvement at diagnosis & $0(0 \%)$ & $13(7.8 \%)$ & \\
\hline \multicolumn{4}{|l|}{ Frontline Therapy regimen } \\
\hline $\mathrm{R}-\mathrm{CHOP}$ & $19(86.4 \%)$ & $130(78.4 \%)$ & \\
\hline R-Benda & $0(0 \%)$ & $16(9.6 \%)$ & \\
\hline R-Tro & $2(9.1 \%)$ & $7(4.2 \%)$ & \\
\hline GMALL-B-NHL 2002 & $0(0 \%)$ & $2(1.2 \%)$ & \\
\hline Others & $1(4.5 \%)$ & $8(4.8 \%)$ & \\
\hline \multicolumn{4}{|l|}{ Concurrent low-grade histology } \\
\hline Follicular 1-3A & $18(81.8 \%)$ & - & \\
\hline MZL & $4(18.2 \%)$ & - & \\
\hline
\end{tabular}

DLBCL, diffuse large B-cell Lymphoma; ss, simultaneous secondary; Yrs., years; CNS, central nervous system; LDH, Lactate dehydrogenase; ECOG; Eastern cooperative oncology group; PS, performance status; R, rituximab; CHOP, cyclophosphamide, doxorubicin, vincristine, prednisolone; Tro, Trofosfamide; Benda, Bendamustine, GMALL, German Multicenter Study Group on Adult Acute Lymphoblastic Leukemia B-NHL2002 Protocol; MZL, marginal zone lymphoma; Others, treatment refusal, other rituximab based regimen (e.g. R-Fludarabine+Cyclofosfamide) or palliative cytoreductive treatment. 
of recently demonstrated non-superiority of rituximab maintenance compared to no maintenance therapy following R-CHOP or R-CHOP-like treatment in de novo DLBCL [14, 23].

The most recent study by Magnano et al., published during the preparation of this manuscript, was the first to characterize cases of FL/DLBCL type ssDLBCL cases with regard to Cell of origin (COO) and to explore recurrent chromosomal aberrations (cMYC, BCL2 \& $B C L 6$ ) as well as NOTCH 1/2 mutations [13]. In keeping with published studies higher grade follicular lymphoma (IIIA/B) components were represented more pronounced within the group of follicular lymphoma-type ssDLBCL (13/18 cases $72.2 \%$ ) [24]. Moreover, we found our subsequent observations from FL-type ssDLBCL to be widely in keeping with results published for both de novo

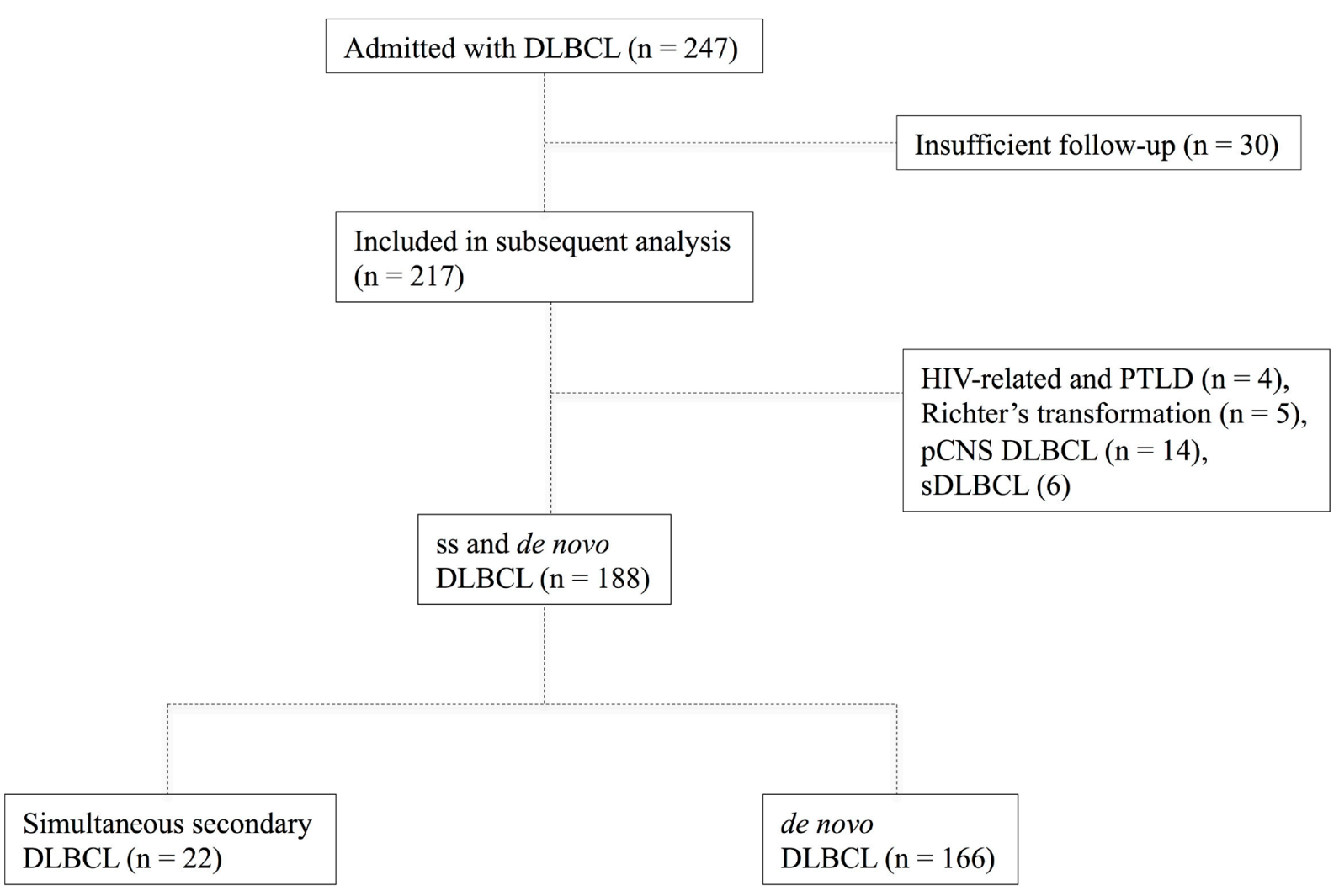

Figure 1: Flowchart depicting the composition of the study group and controls (de novo DLBCL).

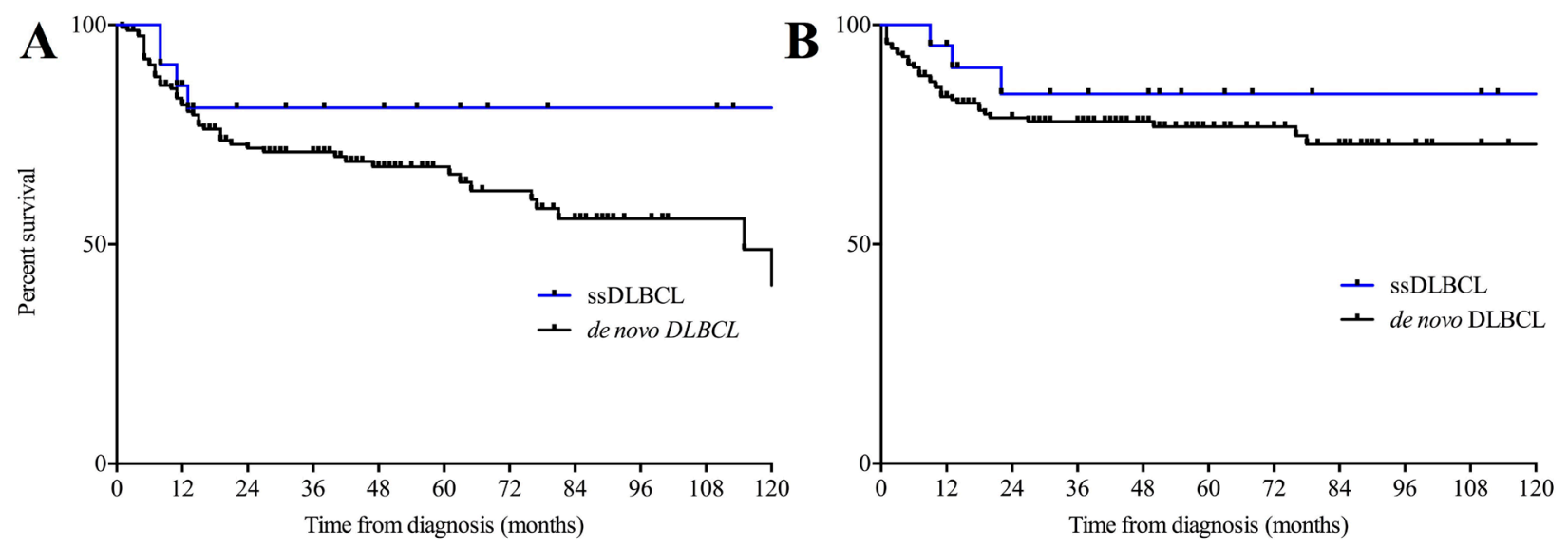

Figure 2: (A) Progression-free and overall (B) survival in simultaneous secondary DLBCL and de novo DLBCL. DLBCL, diffuse large B-cell Lymphoma; ss, simultaneous secondary. 
as well as ssDLBCL regarding COO whereas cases with marginal zone derived indolent lymphoma component showed a predominance of non-GCB origin [13, 25]. In addition we found a statistical trend towards a better clinical outcome among GCB-type ssDLBCL patients, which however failed to reach statistical significance in light of the small study group [13, 26]. Intriguingly, we were however able to detect an impact of underlying indolent lymphoma histology on both OS and PFS favoring follicular lymphoma-type composite disease, which reached statistical significance even within this small study group. Larger and ideally prospective trails will need to address this issue further in order to assess the necessity of treatment intensification (e.g. ASCT) in this small yet non-negligible subgroup of ssDLBCL patients.

The essential conclusion to be drawn from this study is that standard DLBCL-type immunocheotherapy (e.g. R-CHOP) induces a non-inferior response in ssDLBCL compared to de novo DLBCL while therapeutic intensification in the front-line setting appears expendable. To the best of our knowledge this is the first investigation to show that this may however not be the case in composite lymphoma with a non-follicular-type indolent component.
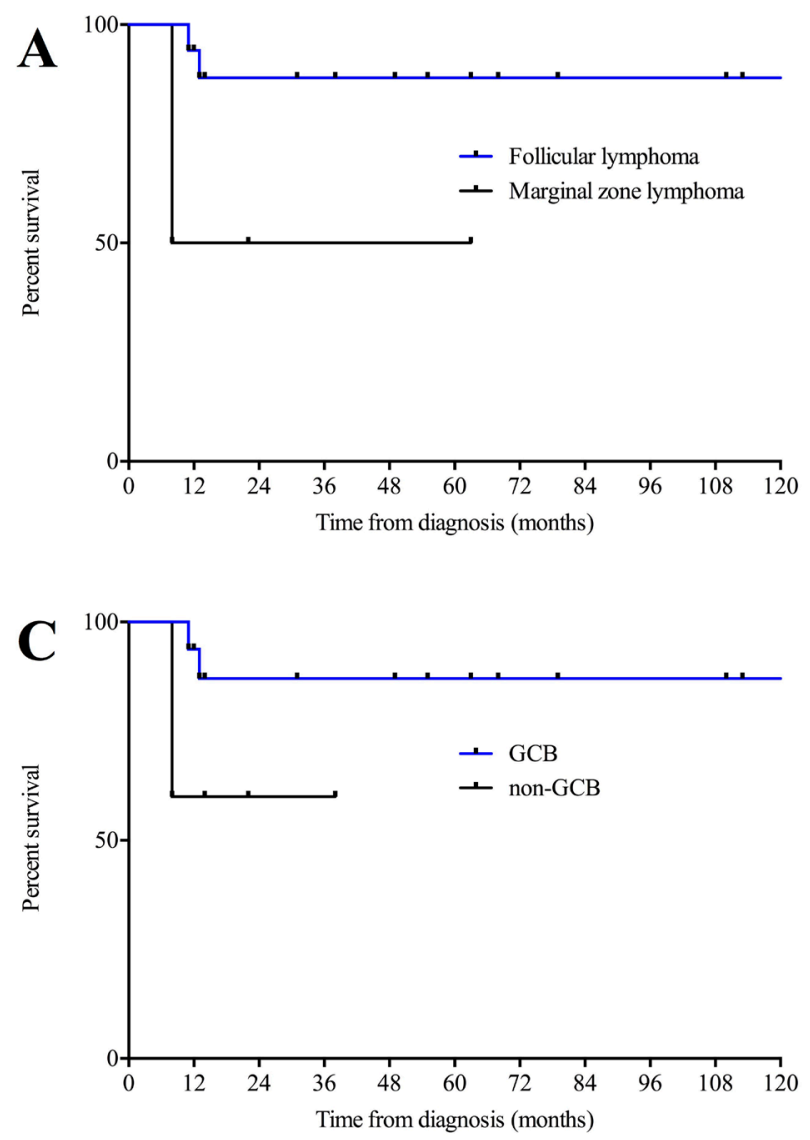

Complementarily, we found no significant rate of double-expressor or double-hit lymphoma. In the light of the newly shaped entity of high-grade B-cell lymphoma with $M Y C, B C L 2$ and or BCL6 aberrations, which was excluded from the current study, however, this was to be expected. The impact of these observations is however diminished again by the limited size of the study group. Another point of interest for future prospective studies would be a systematical comparison of ssDLBCL with transformed indolent lymphoma with a well-documented preceding course of the underlying indolent lymphoma ("late transformations"), which are believed to arise y diverging genomic pathways [27-29]. As only few of these cases were present in our current cohort such an analysis was omitted $(n=6$; data not shown).

Our observation regarding the predominance of highgrade morphology without composite indolent fractions upon relapse may partially be attributed to the limited time of follow-up as recent studies revealed a prolonged progression-free survival in both transformed and nontransformed FL patients in the immunochemotherapy era and the vast majority of ssDLBCL patients in our study received $\mathrm{R}-\mathrm{CHOP}$ induction treatment [6]. As indolent
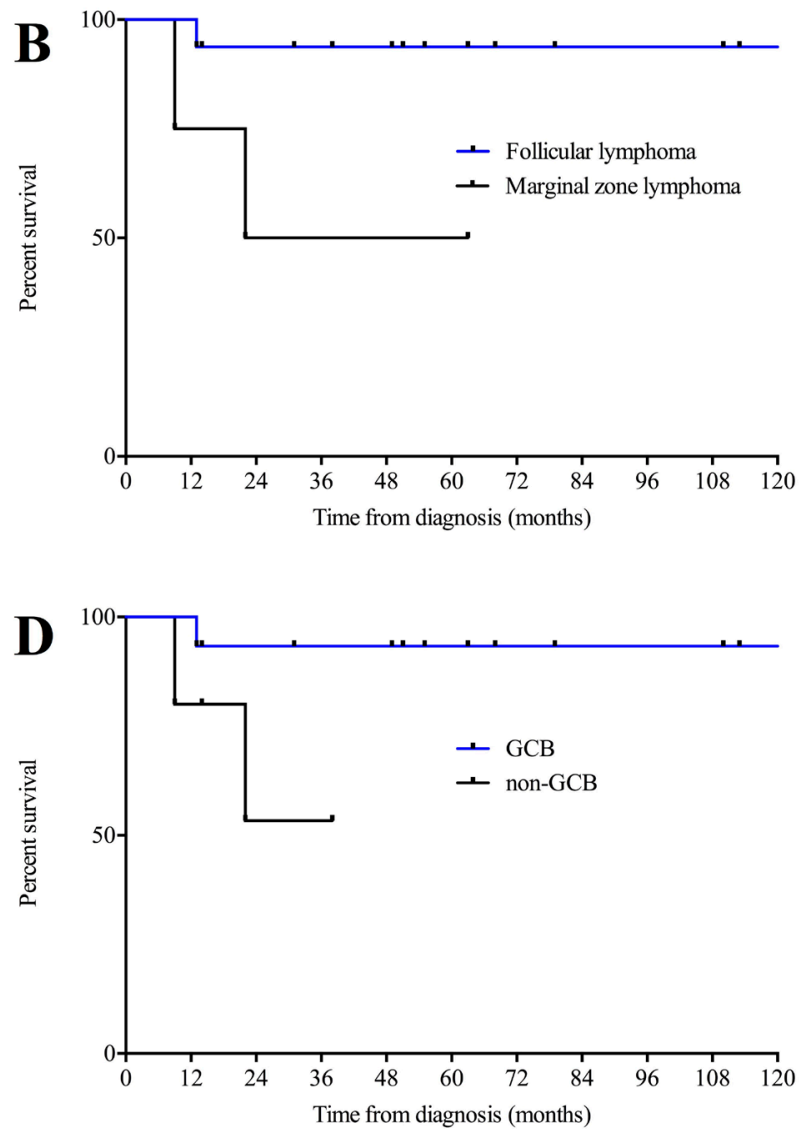

Figure 3: (A) Progression-free and overall (B) survival in ssDLBCL with underlying indolent lymphoma of follicular lymphoma-type and marginal zone lymphoma-type. (C) Progression-free and overall (D) survival in simultaneous secondary DLBCL of GCB and non-GCB type. GCB, germinal center B-cell like. 
lymphoma such as FL have to be considered incurable even in the immunochemotherapy era a subsequent accumulation of relapse derived from the indolent fraction of the disease still is to be expected in our cohort over time although recent studies suggested a reduced rate of such adverse events in the era of immunochemotherapy [18]. Intensive long-term follow-up for these patients is therefore clearly warranted.

Limitations of the present study primarily include its limited sample size, especially relativizing our sub group analysis among ssDLBCL cases based upon histology of the underlying lymphoma. The statistical significances we derived from our data need to be validated in larger and if possible prospective trails before definitive suggestions regarding personalized risk-assessment and ultimately treatment intensifications should be made. Other deficits are principally those typically associated with retrospective studies. Baseline clinical characteristics are derived from the information in the medical records, which harbors a potential for fragmentary data, especially while the vast majority of deaths within the study group were lymphoma related, the fraction of patients lost to follow-up due to nonlymphoma related death can not be securely estimated from our data. Another shortcoming inherent to the retrospective design of the study is the potential of a selection bias of indistinct extent. This aspect is of particular interest in such a limited study group and the current data need therefore to be interpreted as preliminary.

\section{CONCLUSION}

In summary, our findings underline the close clinicopathological relationship between ssDLBCL and de novo DLBCL advocating intensive R-CHOP-like treatment upon initial diagnosis. Moreover both the shortas well as the intermediate-term course of the disease is predominantly determined by the high-grade component with a trend towards a more favorable outcome among germinal center derived ssDLBCL and those with a concurrent follicular lymphoma-type component. A non-FL-type composite DLBCL may however require treatment intensification.

\section{MATERIALS AND METHODS}

\section{Patients}

To evaluate clinical and prognostic characteristics, we reviewed our databases to identify patients with ssDLBCL admitted to our institution (University Hospital Schleswig Holstein Campus Luebeck - covering the population in the area of southern Schleswig Holstein consisting of approximately 1.000. 000 inhabitants) between January 2010 and July 2017, excluding patients with insufficient follow-up (30 patients referred to other centers following primary diagnosis and subsequent loss of follow-up), primary central nervous system (pCNS) DLBCL $(\mathrm{n}=14)$, PTLD and HIV-related Lymphoma $(\mathrm{n}=4)$, Richter's transformation as well as transformed indolent lymphoma $(\mathrm{n}=5 \& 6$ respectively). Clinical information was collected from the original files, and data concerning performance status (Eastern Cooperative Oncology Group [ECOG]), disease extent, treatment modalities, response rate, relapse pattern, cause of death and survival were recorded, as were initial serum levels of lactate dehydrogenase (LDH). Staging was performed according to the Cotswold modifications of the Ann Arbor classification [30]. Moreover all patients were assessed according to the revised international prognostic index (R-IPI) [31].

\section{Centralized histological assessment and criteria for ssDLBCL}

247 consecutive patients with DLBCL (doublehit lymphomas were excluded) diagnosed in accordance with the latest edition of the WHO classification of tumors of the lymphoid system (Reference center for haematopathology, University Hospital of SchleswigHolstein, Campus Luebeck) treated at our institution were identified over the course of the above mentioned time period [3].

The histological criteria for ssDLBCL were defined as the presence of a variable DLBCL component in the lymph node biopsy (both open lymph node excision biopsy as well as needle core biopsy samples were included) otherwise showing FL or other indolent lymphoma infiltrates. By WHO recommendations, a composite histology component was defined as sheets of large cells infiltrating the underlying indolent lymphoma and lacking follicular architecture defined by the absence of dendritic cells in cases of underlying FL (CD23). In cases of underlying marginal-zone-derived indolent lymphoma the populations of small and large B-cells were more diffusely mixed and therefore the diagnosis of ssDLBCL was only established in cases where a concurrent yet sufficiently definable indolent lymphoma component could be proven both morphologically as well as immunophenotypically as described [32]. The percentage of the DLBCL component within the diagnostic biopsy was quantified and cell of origin (COO) was assessed employing the immunohistochemical algorithm described by Hans el al. [33]. Histological slides were reviewed by HM and $\mathrm{NG}$ in order to confirm diagnosis. Antibodies used are summarized in Supplementary Table 1.

Fluorescence-in-situ-hybridization (FISH) for chromosomal aberrations affecting BCL2 / BCL6 / cMYC was not routinely performed but reserved for cases with suspicious morphology and high proliferative activity $($ Ki67 > 90\%). 


\section{Treatment, response assessment, staging and outcome}

Following base line staging investigations according to standard procedures patients were treated with an (immuno-) chemotherapy regimen of the treating physician's choice with R-CHOP-like immunochemotherapy serving as the institutional standard [34]. Bone marrow aspirates (+FACS) and trephine biopsies were routinely performed at initial diagnosis and thereafter in case of prior involvement or clinical suspicion of bone marrow infiltration.

Treatment response was evaluated in accordance with widely established criteria and definitions of complete (CR) and partial response (PR) as well as the method for calculation of overall (OS) and progression free survival (PFS) were employed according to standard definitions [34]. FDG-PET-Scans were not employed on a routine basis for all patients.

\section{Ethics statement}

Written informed consent for retrospective analysis of their clinical data and scientific use of biopsy material was obtained from all patients and the study was approved by the ethics committee of the University of Luebeck (reference-no 17-266).

\section{Statistics}

Time to progression and overall survival (PFS, OS) were calculated from the date of initial diagnosis. Survival (PFS and OS) was primarily estimated by means of the Kaplan-Meier method and the univariate log-rank test. Characteristics found to be associated with either OS or PFS with at least a trend towards statistical significance $(\mathrm{p}<0.07)$ were included in a subsequent multivariate proportional hazard model (Cox proportional hazard) alongside composite histology. Given the non-matched composition of the ssDLBCL and de novo DLBCL groups we conducted a confirmatory propensity score matching approach (matching for age, sex, ECOG, LDH, Stage, $\mathrm{R}-\mathrm{IPI}$ ) resulting in two matched groups (ssDLBCL and de novo DLBCL; $\mathrm{n}=22$ each) followed again by a univariate log-rank test for difference in PFS and OS with regard to the presence of composite histology. Differences among patient subgroups were assessed by using the chi- square as well as the Mann-Whitney-U-test was used when appropriate. All statistical investigations were conducted using GraphPad PRISM 6 and SPSS 24 (IBM).

\section{Author contributions}

Study concept: NG, HW. Data collection: NG, HW, HL, HB, HM. Data analysis and creation of figures and tables: NG, DR, HB, HL. Initial Draft of manuscript: NG. Critical revision and approval of final version: all authors.

\section{ACKNOWLEDGMENTS}

The authors would like to thank Birte Mergelkuhl and Rudina Marx for their dedicated administrative assistance.

\section{CONFLICTS OF INTEREST}

The authors declare no conflicts of interest.

\section{REFERENCES}

1. Qazi R, Aisenberg AC, Long JC. The natural history of nodular lymphoma. Cancer. 1976; 37:1923-1927.

2. Cullen MH, Lister TA, Brearley RI, Shand WS, Stansfeld AG. Histological transformation of non-Hodgkin's lymphoma: a prospective study. Cancer. 1979; 44:645-651.

3. Swerdlow SH, Campo E, Pileri SA, Harris NL, Stein H, Siebert R, Advani R, Ghielmini M, Salles GA, Zelenetz AD, Jaffe ES. The 2016 revision of the World Health Organization classification of lymphoid neoplasms. Blood. 2016; 127:2375-2390.

4. Yuen AR, Kamel OW, Halpern J, Horning SJ. Long-term survival after histologic transformation of low-grade follicular lymphoma. J Clin Oncol. 1995; 13:1726-1733.

5. Bastion Y, Sebban C, Berger F, Felman P, Salles G, Dumontet C, Bryon PA, Coiffier B. Incidence, predictive factors, and outcome of lymphoma transformation in follicular lymphoma patients. J Clin Oncol. 1997; 15:1587-1594.

6. Link BK, Maurer MJ, Nowakowski GS, Ansell SM, Macon WR, Syrbu SI, Slager SL, Thompson CA, Inwards DJ, Johnston PB, Colgan JP, Witzig TE, Habermann TM, Cerhan JR. Rates and outcomes of follicular lymphoma transformation in the immunochemotherapy era: a report from the University of Iowa/MayoClinic Specialized Program of Research Excellence Molecular Epidemiology Resource. J Clin Oncol. 2013; 31:3272-3278.

7. Conconi A, Ponzio C, Lobetti-Bodoni C, Motta M, Rancoita PM, Stathis A, Moccia AA, Mazzucchelli L, Bertoni F, Ghielmini M, Cavalli F, Zucca E. Incidence, risk factors and outcome of histological transformation in follicular lymphoma. Br J Haematol. 2012; 157:188-196.

8. de Jong D, de Boer JP. Predicting transformation in follicular lymphoma. Leuk Lymphoma. 2009; 50:1406-1411.

9. Montoto S, Lopez-Guillermo A, Altes A, Perea G, Ferrer A, Camos M, Villela L, Bosch F, Esteve J, Cervantes F, Blade J, Nomdedeu B, Campo E, et al. Predictive value of Follicular Lymphoma International Prognostic Index (FLIPI) in patients with follicular lymphoma at first progression. Ann Oncol. 2004; 15:1484-1489.

10. Villa D, Crump M, Panzarella T, Savage KJ, Toze CL, Stewart DA, MacDonald DA, Buckstein R, Lee C, 
Alzahrani M, Rubinger M, Foley R, Xenocostas A, et al. Autologous and allogeneic stem-cell transplantation for transformed follicular lymphoma: a report of the Canadian blood and marrow transplant group. J Clin Oncol. 2013; 31:1164-1171.

11. Sarkozy C, Trneny M, Xerri L, Wickham N, Feugier P, Leppa S, Brice P, Soubeyran P, Gomes Da Silva M, Mounier C, Offner F, Dupuis J, Caballero D, et al. Risk Factors and Outcomes for Patients With Follicular Lymphoma Who Had Histologic Transformation After Response to First-Line Immunochemotherapy in the PRIMA Trial. J Clin Oncol. 2016; 34:2575-2582.

12. Reddy N, Oluwole O, Greer JP, Goodman S, Engelhardt B, Jagasia MH, Savani BN. Superior long-term outcome of patients with early transformation of non-Hodgkin lymphoma undergoing stem cell transplantation. Clin Lymphoma Myeloma Leuk. 2012; 12:406-411.

13. Magnano L, Balague O, Dlouhy I, Rovira J, Karube K, Pinyol M, Rivas-Delgado A, Costa D, Martinez-Trillos A, Gonzalez-Farre B, Martinez-Pozo A, Gine E, Colomer D, et al. Clinicobiological features and prognostic impact of diffuse large B-cell lymphoma component in the outcome of patients with previously untreated follicular lymphoma. Ann Oncol. 2017; 28:2799-2805.

14. Bech RS, Nielsen KL, Larsen TS, Bentzen HH, Lynggaard LS, Do TH, Braendstrup P, Brady J, Mikhaeel NG, Dybkaer K, Johnsen HE, Jensen P, Bogsted M, El-Galaly TC. Real world data on rituximab maintenance therapy after frontline immunochemotherapy in grade 1-3a follicular lymphoma. Br J Haematol. 2017 May 23. [Epub ahead of print].

15. Ziepert M, Hasenclever D, Kuhnt E, Glass B, Schmitz N, Pfreundschuh M, Loeffler M. Standard International prognostic index remains a valid predictor of outcome for patients with aggressive CD20+ B-cell lymphoma in the rituximab era. J Clin Oncol. 2010; 28:2373-2380.

16. Dreyling M, Ghielmini M, Rule S, Salles G, Vitolo U, Ladetto M; ESMO Guidelines Committee. Newly diagnosed and relapsed follicular lymphoma: ESMO Clinical Practice Guidelines for diagnosis, treatment and follow-up. Ann Oncol. 2017; 28:3109.

17. Wagner-Johnston ND, Link BK, Byrtek M, Dawson KL, Hainsworth J, Flowers CR, Friedberg JW, Bartlett NL. Outcomes of transformed follicular lymphoma in the modern era: a report from the National LymphoCare Study (NLCS). Blood. 2015; 126:851-857.

18. Al-Tourah AJ, Gill KK, Chhanabhai M, Hoskins PJ, Klasa RJ, Savage KJ, Sehn LH, Shenkier TN, Gascoyne RD, Connors JM. Population-based analysis of incidence and outcome of transformed non-Hodgkin's lymphoma. J Clin Oncol. 2008; 26:5165-5169.

19. Montoto S, Davies AJ, Matthews J, Calaminici M, Norton AJ, Amess J, Vinnicombe S, Waters R, Rohatiner AZ, Lister TA. Risk and clinical implications of transformation of follicular lymphoma to diffuse large B-cell lymphoma. J Clin Oncol. 2007; 25:2426-2433.
20. Acker B, Hoppe RT, Colby TV, Cox RS, Kaplan HS, Rosenberg SA. Histologic conversion in the non-Hodgkin's lymphomas. J Clin Oncol. 1983; 1:11-16.

21. Morley NJ, Evans LS, Goepel J, Hancock BW. Transformed follicular lymphoma: the 25-year experience of a UK provincial lymphoma treatment centre. Oncol Rep. 2008; 20:953-956.

22. Hans CP, Weisenburger DD, Vose JM, Hock LM, Lynch JC, Aoun P, Greiner TC, Chan WC, Bociek RG, Bierman PJ, Armitage JO. A significant diffuse component predicts for inferior survival in grade 3 follicular lymphoma, but cytologic subtypes do not predict survival. Blood. 2003; 101:2363-2367.

23. Epperla N, Pham AQ, Burnette BL, Wiseman GA, Habermann TM, Macon WR, Ansell SM, Inwards DJ, Micallef IN, Johnston PB, Markovic SN, Porrata LF, Colgan JP, et al. Risk of histological transformation and therapy-related myelodysplasia/acute myeloid leukaemia in patients receiving radioimmunotherapy for follicular lymphoma. Br J Haematol. 2017; 178:427-433.

24. Wahlin BE, Yri OE, Kimby E, Holte H, Delabie J, Smeland EB, Sundstrom C, Christensson B, Sander B. Clinical significance of the WHO grades of follicular lymphoma in a population-based cohort of 505 patients with long follow-up times. Br J Haematol. 2012; 156:225-233.

25. Alizadeh AA, Eisen MB, Davis RE, Ma C, Lossos IS, Rosenwald A, Boldrick JC, Sabet H, Tran T, Yu X, Powell JI, Yang L, Marti GE, et al. Distinct types of diffuse large B-cell lymphoma identified by gene expression profiling. Nature. 2000; 403:503-511.

26. Rosenwald A, Wright G, Chan WC, Connors JM, Campo E, Fisher RI, Gascoyne RD, Muller-Hermelink HK, Smeland EB, Giltnane JM, Hurt EM, Zhao H, Averett L, et al. The use of molecular profiling to predict survival after chemotherapy for diffuse large-B-cell lymphoma. N Engl J Med. 2002; 346:1937-1947.

27. Okosun J, Montoto S, Fitzgibbon J. The routes for transformation of follicular lymphoma. Curr Opin Hematol. 2016; 23:385-391.

28. Pasqualucci L, Khiabanian H, Fangazio M, Vasishtha M, Messina M, Holmes AB, Ouillette P, Trifonov V, Rossi D, Tabbo F, Ponzoni M, Chadburn A, Murty VV, et al. Genetics of follicular lymphoma transformation. Cell Rep. 2014; 6:130-140.

29. Ruminy P, Jardin F, Picquenot JM, Parmentier F, Contentin $\mathrm{N}$, Buchonnet G, Tison S, Rainville V, Tilly H, Bastard C. S(mu) mutation patterns suggest different progression pathways in follicular lymphoma: early direct or late from FL progenitor cells. Blood. 2008; 112:1951-1959.

30. Lister TA, Crowther D, Sutcliffe SB, Glatstein E, Canellos GP, Young RC, Rosenberg SA, Coltman CA, Tubiana M. Report of a committee convened to discuss the evaluation and staging of patients with Hodgkin's disease: Cotswolds meeting. J Clin Oncol. 1989; 7:1630-1636. 
31. Sehn LH, Berry B, Chhanabhai M, Fitzgerald C, Gill K, Hoskins P, Klasa R, Savage KJ, Shenkier T, Sutherland J, Gascoyne RD, Connors JM. The revised International Prognostic Index (R-IPI) is a better predictor of outcome than the standard IPI for patients with diffuse large B-cell lymphoma treated with R-CHOP. Blood. 2007; 109:1857-1861.

32. Ghesquieres H, Berger F, Felman P, Callet-Bauchu E, Bryon PA, Traverse-Glehen A, Thieblemont C, Baseggio L, Michallet AS, Coiffier B, Salles G. Clinicopathologic characteristics and outcome of diffuse large B-cell lymphomas presenting with an associated low-grade component at diagnosis. J Clin Oncol. 2006; 24:5234-5241.
33. Hans CP, Weisenburger DD, Greiner TC, Gascoyne RD, Delabie J, Ott G, Muller-Hermelink HK, Campo E, Braziel RM, Jaffe ES, Pan Z, Farinha P, Smith LM, et al. Confirmation of the molecular classification of diffuse large B-cell lymphoma by immunohistochemistry using a tissue microarray. Blood. 2004; 103:275-282.

34. Cheson BD, Pfistner B, Juweid ME, Gascoyne RD, Specht L, Horning SJ, Coiffier B, Fisher RI, Hagenbeek A, Zucca E, Rosen ST, Stroobants S, Lister TA, et al. Revised response criteria for malignant lymphoma. J Clin Oncol. 2007; 25:579-586. 